\title{
A BOUND FOR THE CLASS OF GERTAIN NILPOTENT GROUPS
}

\author{
CHANDER KANTA GUPTA *
}

(Received 4 April 1965)

\section{Introduction}

The groups whose 2-generator subgroups are all nilpotent of class at most 2 are nilpotent of class at most 3 (see Levi [6]). Heineken [3] generalized Levi's result by proving that for $n \geqq 3$, if the $n$-generator subgroups of a group are all nilpotent of class at most $n$, then the group itself is nilpotent of class at most $n$. Other related problems have been considered by Bruck [1].

Another problem of similar interest is to seek information about the groups all of whose proper subgroups are nilpotent of class at most $n(n \geqq 1)$. It is known that the group itself need not be nilpotent at all. Finite nonnilpotent groups all of whose proper subgroups are nilpotent have been studied in detail by Iwasawa [4] and Rédei [10]. Newman and Wiegold [8] have considered infinite non-nilpotent groups with the above property. If, however, a group $G$ is nilpotent and has all its proper subgroups of class at most $n$, then by $[2, \mathrm{p} .153]$ the class of $G$ cannot exceed $2 n$ and, at least for certain special values of $n$, it is known that there are such groups with class precisely $2 n$ (c.f. Rédei [9] when $n=1$ and Macdonald [7] when $\boldsymbol{n}=3$ ). The main result of this paper is contained in the following theorem.

THEOREM 1.1. Let $n$ and $d$ be positive integers greater than 1. If $G$ is a nilpotent group whose proper subgroups are all nilpotent of class at most $n$. then the class of $G$ is at most $m$, where $m \leqq(n d / d-1)<m+1$ and $d$ is the minimal number of generators of $G$.

The other two theorems proved in this paper are,

THEOREM 1.2. If $G$ is a nilpotent group whose proper subgroups are all of class at most $n$, then $G$ has class at most $n$ or $G$ is a p-group for some prime $p$.

THEOREM 1.3. Let $n$ be an integer greater than 2. If $G$ is a finite metabelian nilpotent group all of rohose proper subgroups are of class at most $n$ and if $G$ is minimally generated by $n$ elements, then $G$ has class at most $n$ or $G$ is a 2-group.

- This work is a part of my dissertation presented for the degree of Master of Arts at the Australian National University, Canberra. 
If $n=d=2$, then by Theorem 1.1, $G$ has class at most 4 . This, however, is not the best possible bound since it has been proved by Macdonald [7], Kappe [5] (and the author independently), that in this case the class of $G$ is at most 3.

If $n=d \geqq 3$, then by Theorem $1.1, G$ has class at most $n+1$. The last section of this paper is devoted to exhibiting groups of class precisely $n+1$ which are minimally generated by $n$ elements and whose proper subgroups are all of class at most $n$. This shows that the bound given by Theorem 1.1 is best possible when $n=d \geqq 3$.

I thank my supervisor Dr M. F. Newman for suggesting the topic of this research and for his general guidance. I also thank Dr. L. G. Kovács for his many useful suggestions. My thanks are also given to Professors B. H. Neumann and A. Brown for providing me the opportunity to do this work.

\section{Definitions and notations}

We write $a^{b}=b^{-1} a b$. The commutator $[a, b]$ of $a$ and $b$ is $a^{-1} b^{-1} a b$ and, for $n>2$,

$$
\left[a_{1}, a_{2}, \cdots, a_{n}\right]=\left[\left[a_{1}, a_{2}, \cdots, a_{n-1}\right], a_{n}\right]
$$

defines a left-normed commutator of weight $n$.

If $A$ and $B$ are subgroups of $G$, then $[\dot{A}, B]$ is defined to be the subgroup of $G$ generated by the commutators $[a, b]$ where $a \in A$ and $b \in B$. In particular, the subgroup $[G, G]$ is called the derived group of $G$.

The normal series

where

$$
G=\gamma_{1}(G) \geqq \gamma_{2}(G) \geqq \gamma_{3}(G) \geqq \cdots
$$

$$
\gamma_{i+1}(G)=\left[\gamma_{i}(G), G\right]
$$

is called the lower central series of $G$. In particular $\gamma_{2}(G)$ is the derived group of $G$. If $\gamma_{n+1}(G)=1$ then $G$ is said to be nilpotent of class at most $n$.

The normal series

$$
1=Z_{0}(G) \leqq Z_{1}(G) \leqq Z_{2}(G) \leqq \cdots
$$

where $Z_{1}(G)$ is the centre of $G$ and

$$
Z_{i+1}(G) / Z_{i}(G)=Z_{1}\left(G / Z_{i}(G)\right)
$$

is called the upper central series of $G$.

Let $a, b, c$ be arbitrary elements of a group $G$, then the following commutator identities are standard and will be used without reference: 


$$
\begin{gathered}
{[a b, c]=[a, c]^{b}[b, c] .} \\
{[a, b c]=[a, c][a, b]^{e} .} \\
{\left[a, b^{-1}, c\right]^{b}\left[b, c^{-1}, a\right]^{c}\left[c, a^{-1}, b\right]^{a}=1 .}
\end{gathered}
$$

A direct consequence of the last identity is the following identity:

$$
[a, b, c][c, a, b][b, c, a] \in \gamma_{4}(G) \text {. }
$$

\section{Proof of the theorem 1.1}

First we prove the following

LEMMA 3.1. Let $H$ be a normal subgroup of a group $G$. If $\left\{g_{1}, g_{2}, \cdots g_{n}\right\}$ is a family of elements of $G$ which contains $m$ elements of $H(m \leqq n)$, then $\left[g_{1}, g_{2}, \cdots, g_{n}\right] \in \gamma_{m}(H)$.

Proof. The proof is by induction on $m$. If $m=1$, the lemma is trivial. Let $m$ be greater than 1 and suppose the result is true for all positive integers less than $m$. Consider the commutator $\left[g_{1}, g_{2}, \cdots, g_{n}\right]$. If $g_{n} \in H$, then $\left[g_{1}, g_{2}, \cdots, g_{n-1}\right]$ contains at least $m-1$ entries from $H$ and so by the induction hypothesis it belongs to $\gamma_{m-1}(H)$. Therefore,

$$
\left[g_{1}, g_{2}, \cdots, g_{n}\right] \in\left[\gamma_{m-1}(H), H\right]=\gamma_{m}(H) .
$$

If $g_{n} \notin H$, then $\left[g_{1}, g_{2}, \cdots g_{n-1}\right]$ has already at least $m$ entries from $H$ and, therefore, it belongs to $\gamma_{m}(H)$. Hence $\left[g_{1}, g_{2}, \cdots, g_{n}\right] \in\left[\gamma_{m}(H), G\right]$ $\leqq \gamma_{m}(H)$, since $\gamma_{m}(H)$ is normal in $G$. This completes the proof of the lemma.

The following lemma can be easily proved.

LEMMA 3.2. Let $X$ denote a set of generators of a group $G$. If the commutator $\left[x_{1}, x_{2}, \cdots, x_{n}\right]$ is equal to 1 whenever $x_{1}, x_{2}, \cdots, x_{n} \in X$, then $G$ is nilpotent of class at most $n-1$.

To prove Theorem 1.1 , let $X=\left\{x_{1}, x_{2}, \cdots x_{d}\right\}$ be a set of generators of $G$. To show that $G$ has lcass at most $m$, it is sufficient, by Lemma 3.2, to show that an arbitrary commutator $\left[y_{1}, y_{2}, \cdots, y_{m+1}\right]$ is equal to 1 , where each $y_{i} \in X$. Since $m=n+l$ where $l \leqq(n / d-1)<l+1$, we have that $(l+1) d>m+1$. This implies that not all the elements of $X$ can occur more than $l$ times in $\left[y_{1}, y_{2}, \cdots, y_{m+1}\right]$. Thus, there is an element, say $x_{1}$, which occurs at most $l$ times in this commutator.

Since, $x_{2}, x_{3}, \cdots x_{d}$ do not generate $G$, there is a maximal subgroup of $G$, call it $H$, which contains $x_{2}, x_{3}, \cdots, x_{a}$. By Corollary 10.3.2 of [2], $H$ is normal in $G$. Now, $\left[y_{1}, y_{2}, \cdots, y_{m+1}\right]$ contains at least $m+1-l=n+1$ entries from $H$. Thus by Lemma 3.1, $\left[y_{1}, y_{2}, \cdots, y_{m+1}\right] \in \gamma_{m+1}(H)=E$ since $H$ is proper in $G$. This completes the proof of the Theorem 1.1. 
The following are immediate corollaries of Theorem 1.1.

COROLLARY 3.3. If $G$ is a nilpotent group whose proper subgroups are all of class at most $n$, then either $G$ has class at most $n$ or $G$ can be generated by $n+1$ elements.

CoROLLARY 3.4. If $G$ is nilpotent of class $2 n$ and if the proper subgroups of $G$ are all nilpotent of class at most $n$, then $G$ can be generated by 2 elements.

Proof of the Theorem 1.2. First we quote the following

LEMMA 3.5. ([8] Theorem 3.3). If $G$ is an infinite nilpotent group whose proper subgroups are all of class at most $n$, then $G$ has class at most $n$.

To prove Theorem 1.2, let $G$ be of class greater than $n$, then, by Lemma 3.5, $G$ is finite and hence is the direct product of its Sylow subgroups. If there is more than one non-trivial Sylow subgroup, then the class of $G$ is at most $n$; and otherwise $G$ is a $p$-group for some prime $p$.

Proof of the Theorem 1.3. The following lemmas are required.

LEMMA 3.6. (Heineken [3]). If $G$ is a nilpotent group all of whose 3generator subgroups have class at most 3 , then $G$ has class at most 3.

Lemma 3.7. Theorem 1.3 is true for $n=3$.

Proof. If $G$ does not have class at most 3 then, by Theorem 1.1, it has class precisely 4 . Also by Theorem $1.2, G$ is a $p$-group for some prime $p$. Since every 2-generator subgroup of $G$ has class at most $3, G$ satisfies the identities,

$$
\begin{aligned}
& {[a, b, b, b]=1, \quad[a, b, a, a]=1 \text {, }} \\
& {[a, b, a, b]=1 \text { and }[a, b, b, a]=1 \text {. }}
\end{aligned}
$$

Also since $\gamma_{5}(G)=E,[a, b c, b c, a]=1,[a, b c, a, b c]=1$ and $[a c, b, a c, b]=1$ give respectively (by using $\mathrm{A}$ ),

$$
\begin{aligned}
{[b, a, c, a]=} & {[a, c, b, a] ; \quad[b, a, a, c]=[a, c, a, b] ; } \\
& {[b, a, c, b]=[c, b, a, b] . }
\end{aligned}
$$

Further, $[a c, b c, a c, b c]=1$ gives (by using $\mathrm{A}$ and $\mathrm{B}$ ),

$$
\begin{aligned}
{[a, b, c, c] } & =[a, c, b, c]^{-1}[a, c, c, b]^{-1} \\
& =[a, c, b, c]^{-2} \quad \text { (since } G \text { is metabelian). }
\end{aligned}
$$

Commuting both sides of 2.1 by $c$ and applying $B$ gives,

$$
[a, b, c, c]=[a, c, b, c]^{2}
$$

which together with $\mathrm{C}$ gives 


$$
[a, c, b, c]^{4}=1, \quad[a, b, c, c]^{2}=1 .
$$

If $p$ is different from 2 , then $E$ gives $[a, c, b, c]=1$ and $[a, b, c, c]=1$ which together with $B$ give that $G$ is nilpotent of class at most 3 , contrary to our assumption. Thus $p=2$ and the lemma is proved.

To prove Theorem $\mathbf{1 . 3}$, it is sufficient to show that if $G$ is not a 2group, then $G / Z_{n-3}(G)$ has class at most 3. Put $J=Z_{n-3}(G)$. Let $a, b \in G$; and consider the commutator $\left[w_{1} J, w_{2} J, w_{3} J, w_{4} J\right]$ in $G / J$ where $w_{i} \in \operatorname{Sgp}\{a, b\}$. Let $a_{1}, a_{2}, \cdots, a_{-3}$ be arbitrary elements of $G$. Since $\operatorname{Sgp}\left\{a, b, a_{1}, a_{2}, \cdots, a_{n-3}\right\}$ is proper in $G$, it has class at most $n$. In particular, $\left[w_{1}, w_{2}, w_{3}, w_{4}, a_{1}, a_{2}, \cdots, a_{n-3}\right]=1$, so that $\left[w_{1}, w_{2}, w_{3}, w_{4}\right] \in J$. Thus Sgp $\{a J, b J\}$ has class at most 3 , that is, every 2-generator subgroup of $G / J$ has class at most 3 .

Suppose that the class of $G / J$ is greater than 3 . Let $H$ be the smallest subgroup of $G / J$ which is of class greater than 3 , then by the above argument, $d(H) \geqq 3$, where $d(H)$ is minimal numbers of generators of $H$. If $d(H)=3$, then, since every proper subgroup of $H$ is of class at most 3, by Lemma 3.7, $H$ is of class at most 3 , contrary to assumption. If $d(H)>3$, then each 3 generator subgroup of $H$ is of class at most 3 ; and by Lemma 3.6, $H$ has class at most 3 , which is again contrary to assumption. Thus the class of $G / J$ is at most 3 , as was required.

\section{Examples}

Example 4.1. Let $p$ be an odd prime. There exists a group $G$ of class precisely 4, minimally generated by 3-elements and whose proper subgroups are all of class at most 3 .

Such a group $G$ is generated by $a, b, c, x_{1}, x_{2}, \cdots, x_{8}$; with the following relations,

$$
\begin{aligned}
& a^{b}=b^{p}=c^{p}=1 ; x_{i}^{p}=1 \text { for } i=1,2, \cdots, 8 ; \\
& {\left[x_{i}, x_{i}\right]=1 \text { for } i, j=2,3, \cdots, 8 ; x_{2}^{x_{1}}=x_{2} x_{8}^{-1},} \\
& x_{i}^{a_{1}}=x_{i} \text { for } i=3,4, \cdots, 8 ; x_{2}^{a}=x_{2} x_{6}, \\
& x_{i}^{a}=x_{i} \text { for } i=3,4, \cdots, 8 ; \\
& a^{b}=a x_{3}, x_{1}^{b}=x_{1} x_{4}, x_{i}^{b}=x_{i} \text { for } i=2,3, \cdots, 8 ; \\
& a^{o}=a x_{1}^{-1}, b^{c}=b x_{2}^{-1}, x_{1}^{c}=x_{1} x_{5}, x_{2}^{c}=x_{2} x_{7}, \\
& x_{3}^{c}=x_{3} x_{4}^{-1} x_{8}^{-1} x_{6}, x_{4}^{c}=x_{4} x_{8}, x_{8}^{o}=x_{6} x_{8}^{-1}, \\
& x_{i}^{o}=x_{i} \text { for } i=5,7,8 .
\end{aligned}
$$

( $G$ can be constructed in the usual way by three splitting extensions.)

Example 4.2. To each integer $n \geqq 4$, there is an $n$-generator group of class precisely $n+1$ whose proper subgroups are all of class at most $n$. 
Consider the set $N=\{1,2,3, \cdots, n\}$ and let $S$ denote the set of all subsets of $N$ excluding the empty set and the set consisting of 1 alone.

Let $X=\operatorname{gp}\left\{x_{a} \mid x_{a}^{2}=\left[x_{a}, x_{a^{\prime}}\right]=1\right.$ for all $\left.s, s^{\prime} \in S\right\}$. This clearly admits pairwise commuting automorphisms $\alpha_{i}(i \in\{2,3, \ldots, n\})$ of order 2 which map $x_{s}$ to $x_{s}$ if $i \in s$ and $x_{g} \cdot x_{s \cup\{i\}}$ if $i \notin s$. Let $B$ be the splitting extension of $X$ by

$$
A=\operatorname{gp}\left\{a_{i} \mid a_{i}^{2}=\left[a_{i}, a_{j}\right]=1 \text { for all } i, j=2,3, \cdots, n\right\},
$$

the $a_{i}$ inducing the automorphisms $\alpha_{i}$ for $i=2,3, \cdots, n$. There is an automorphism $\alpha_{1}$ of order 4 of $B$ which maps $a_{i}$ to $a_{i} x_{\{i\}}$ for $i=2, \cdots, n ; x_{n}$ to $x_{8}$ if $l \in s$ and $x_{8} \cdot x_{8} \cup\{1\}$ if $I \notin s$. The required group $C$ is then the splitting extension of $B$ by the cyclic group $\left\{a_{1}\right\}$ of order $4, a_{1}$ inducing $\alpha_{1}$ on $B$. The verification of the details is tedious though routine and is left to the interested reader.

\section{References}

[1] R. H. Bruck, Engel conditions in groups and related questions. Lecture Notes; Third Summer Research Institute of the Aust. Math. Soc., 1963, Canberra.

[2] M. Hall. Jr., The Theory of Groups, Macmillan, 1959.

[3] H. Heineken, Uber ein Levisches Nilpotenzkriterium, Archiv, der Math. 12 (1961), 176-178.

[4] Kenkiti Iwasawa, Uber die Struktur der endlichen Gruppen, deren echte Untergruppen Sämtlich nilpotent sind. Proc. Phys-Math. Soc. Japan. 23 (1941), 1-4.

[5] Wolfgang Kappe, Gruppen Theoretische Eigenschaften Und Charakteristische Untergruppen, Archiv. der Math. 13 (1962), 38-48.

[6] F. W. Levi, Groups in which the Commutator operation satisfies certain algebraic conditions, J. Indian Math. Soc. (N.S.) 6 (1942) 87-97.

[7] I. D. Macdonald, Generalizations of a classical theorem about nilpotent groups. II. J. Math. 8 (1964), 556-570.

[8] M. F. Newman and James Wiegold, Groups with many nilpotent subgroups, Arch. Math. 15 (1964), 241-250.

[9] L. Rédei, Das „Schiefe Produkt” in der Gruppentheorie mit Anwendung auf die endlichen nichtcommutativen Gruppen mit lauter Kommutativen echten Untergruppen und die Ordnungszahlen, zu denen nur Kommutative Gruppen gehören, Comm. Math. Helv, 20 (1947), 225-264.

[10] Ladislaus Rédei, Die endlichen einstufig nicht nilpotenten Gruppen, Publ. Math. Debrecen 4 (1966), 303-324.

Institute of Advanced Studies

The Australian National University

Canberra. A.C.T. 